\title{
Evaluation of RP2 and RP3 genes in an X-linked RP family manifesting loss of central vision and preserved peripheral function
}

Received: December 1, 2000 / Accepted: January 17, 2001

\begin{abstract}
X-Linked retinitis pigmentosa is a most severe and heterogeneous disorder of the retina. Recently, genes ( $R P 2$ and $R P G R$ ) from two X-linked loci have been positionally cloned and mutations have been identified in many families. To further evaluate allelic and non-allelic heterogeneity and the genotype - phenotype relationships, and to determine the prevalence of mutations in the gene, we have analyzed one previously unreported X-linked retinitis pigmentosa family, using a combination of haplotype analysis and DNA sequencing. Our extensive analysis of the $R P 2$ gene failed to detect any disease - causing or polymorphic mutations. In the case of the RP3 gene, the alleles of the dinucleotide repeat marker did not segregate with the disease. Although we cannot completely exclude the possibility of the $R P 2$ and $R P 3$ genes as candidate genes, the above results suggest that structural and functional changes associated with the $R P 2$ gene are not responsible for the phenotype in the family analyzed. Further identification of the X-linked genes may facilitate the elucidation of the molecular basis of the disorder in the family analyzed.
\end{abstract}

Key words Retinitis pigmentosa - X-linked - Mutation · Linkage $\cdot$ Haplotype

\section{Introduction}

Retinitis pigmentosa (RP) is a group of progressive retinal disorders with autosomal dominant, recessive, and X-linked forms. Of these, the X-linked disorder (XLRP) has consistently been shown to be the most severe type, and affected

M. Hiraoka $\cdot$ B.S. Shastry $(\bowtie)$

Department of Biological Sciences, Oakland University, Rochester, MI 48309, USA

Tel. +1-248-370-2994; Fax +1-248-370-4225

e-mail: Barkur@aol.com

M.T. Trese

William Beaumont Hospital, Royal Oak, MI, USA males manifest an early onset of the disorder with severe myopia. RP is characterized by a loss of the peripheral visual field, and night blindness (Bird 1975). The disease affects both eyes, and the loss of central vision does not usually occur until age 50, although in some X-linked pedigrees, it may occur much earlier. When the disease progresses, loss of central vision occurs with degeneration of the retina, which leads to complete loss of sight. Carrier females usually have normal vision and a normal fundus. More than 25 loci have been mapped by linkage analysis, and, among these, at least two loci for XLRP have been identified on the short arm of the X-chromosome (Teague et al. 1994). These are termed $R P 2$ and $R P 3$. Recently, genes ( $R P 2$ and $R P G R)$ from these two X-linked loci have been positionally cloned (Meindl et al. 1996; Schwahn et al. 1998) and mutations have been identified in many families. To further evaluate allelic and non-allelic heterogeneity, and to establish a relationship between genotype and phenotype, we have analyzed one previously unreported XLRP family for mutations in the $R P 2$ and $R P 3$ genes.

\section{Subjects and methods}

The pedigree used in this study is shown in Fig. 1A and was described previously (Shastry and Trese 1995). All patients were informed of the purpose of the study, which was approved by the Institutional Review Board of Oakland University. Venous blood was collected from several affected and unaffected individuals, and high molecular-weight DNA was extracted as described before (Shastry et al. 1995). For the purpose of DNA analysis, all five exons of the $R P 2$ gene were amplified by the polymerase chain reaction (PCR), using commercially synthesized primers (Schwahn et al. 1998) spanning the splice sites. The PCR products were purified and directly sequenced using a cycle sequencing kit (Applied Biosystems, Foster City, CA, USA), according to the manufacturer's instructions. All samples were sequenced on both strands, using the same primers as for the PCR. 
A

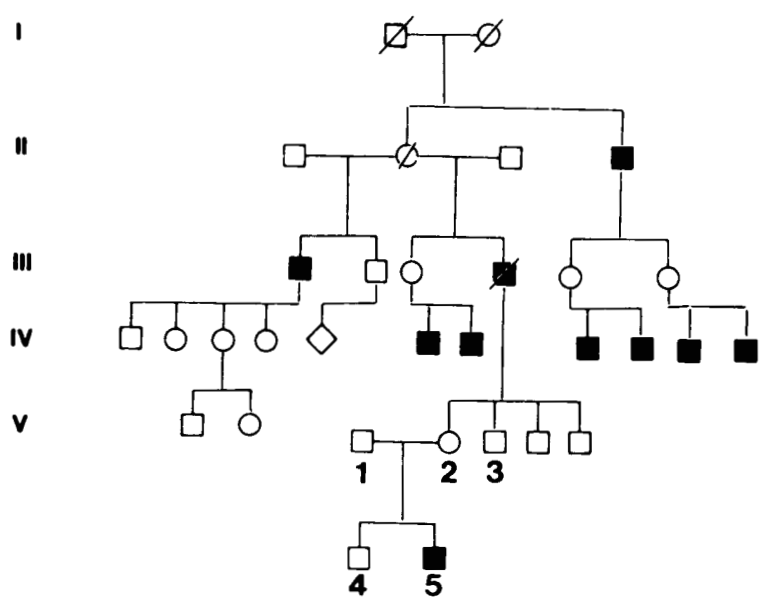

B
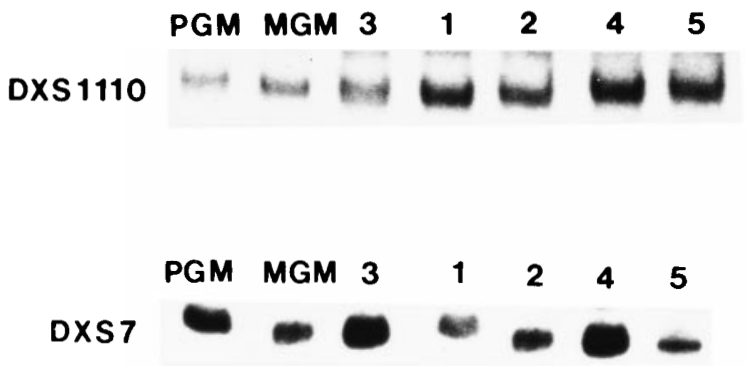

DXS 426

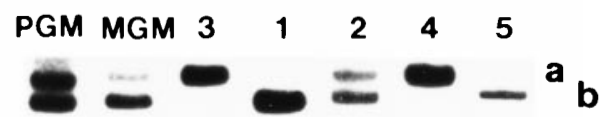

Fig. 1. A Pedigree of a large family in which $\mathrm{X}$-linked retinitis pigmentosa (RP) is segregating. Clinically affected individuals are represented by filled squares. The deceased are marked with a slash. B The segregation of polymerase chain reaction (PCR) alleles identified with the probes DXS $1110(R P 3)$, DXS7, and DXS $426(R P 2)$ is shown. The alleles produced by the PCR at the DXS 426 locus can be followed, and it can be seen that the RP syndrome appears to be transmitted with the $\mathrm{b}$ allele. The numbers 1-5 on the top of the lanes correspond to the numbers $1-5$ in the pedigree. Although the differences in the intensity of bands in lane MGM could be due to the differential amplification of the two alleles, the reason for this difference in intensity is not known. $P G M$, Paternal grandmother; $M G M$, maternal grandmother

\section{Results}

The kindred shown in Fig. 1 has a history of RP. The clinical details (including electroretinography [ERG], visual acuity, and field data) of the family were described previously (Shastry and Trese 1995).The proband has a pigmentary retinopathy similar to RP and high myopia. He has no other medical problems. Interestingly, however, all affected family members exhibited loss of central vision, in contrast to peripheral vision, at the age of 10-12 years. This suggests an unusual, severe cone - rod dystrophy in the family. In the pedigree, there are no male-to-male transmissions; all

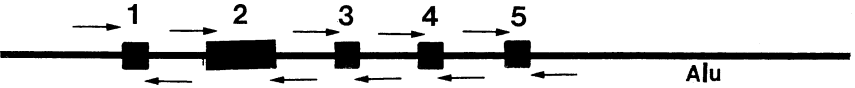

Fig. 2. A schematic representation of the $R P 2$ gene, showing the regions sequenced. The arrows represent the primers. For exon 2, four different overlapping primers were used. The exons are numbered. The figure is not drawn to scale

affected individuals are males and the transmission is from affected man to carrier daughter, to affected grandson, all consistent with an X-linked pattern of inheritance. In order to localize the disease gene and to categorize this XLRP kindred as being an RP2 or RP3 subtype, we first carried out haplotype analysis of markers close to the $R P 2$ and $R P 3$ candidate gene loci. A total of 11 microsatellite markers (DXS 228; DXS 1110; DXS 7; MAOA-3; DXS 426; DXS 453; DXY S1X; DXS 3; DXS 454; DXS 425; and DXS 292) spanning the entire $\mathrm{X}$-chromosome have been analyzed. The results shown in Fig. 1B suggest that DXS 426 marker alleles could be followed and there was segregation of the DXS 426 locus with the disease. Because this marker has previously been shown to be linked to the RP2 type disorder (Coleman et al. 1990), and because several other Xp, as well as Xq markers, could not be followed with the disease, the above results are indicative of evidence for an RP2 subtype disorder in this family. Unfortunately, further linkage analysis could not be undertaken because additional affected and unaffected family members declined to be tested. We next screened the genomic DNA from the proband and other available family members for mutations in the $R P 2$ gene. Our extensive sequence analysis of all five exons (Fig. 2) revealed a wild-type sequence in the entire coding region, as well as splice sites of the $R P 2$ gene. Although we cannot completely rule out the $R P 2$ gene as a candidate gene in the present study (mutations in the upstream promoter or intronic sequence may be implicated), it appears unlikely that the RP2 type pathology in the family is caused by sequence variations in the coding region and splice sites of the $R P 2$ gene.

\section{Discussion}

RP exhibits exceptional genetic and clinical heterogeneity. In XLRP, it has been suggested that RP2 and RP3 represent the two predominant forms (Teague et al. 1994). Mutational analysis of the $R P G R$ and $R P 2$ genes together accounted for approximately $30 \%$ of familial XLRP cases (Hardcastle et al. 1999; Mears et al. 1999) mapped to these genetic loci. This still leaves about $70 \%$ of cases unaccounted for. One possible explanation is that the above results could be due to microheterogeneity around the RP2 and RP3 loci. This possibility is supported by the recent identification of additional XLRP loci (Mears et al. 1999). Our extensive sequence analysis of one RP2 family also failed to detect any mutations in the $R P 2$ gene, suggesting again that the $R P 2$ gene is not the major gene responsible for the RP2 subtype disorder. These results provide additional support 
for microheterogeneity around the $R P 2$ locus, and will be helpful in establishing a relationship between the genotype and phenotype. In the future, identification of the gene responsible for the disorder may provide significant insight into the molecular basis of the disorder in this family. Meanwhile, contributions from genetic studies of other RP families will enrich our understanding of the molecular basis of several subtypes of this complex disorder.

Acknowledgments This work was supported in part by a grant from the Retinopathy of Prematurity (ROPARD) foundation and the Fight for Sight Research Division of Prevent Blindness America.

\section{References}

Bird AC (1975) X-Linked retinitis pigmentosa. Br J Ophthalmol 59:177-199

Coleman M, Bhattacharya SS, Lindsay S, Wright A, Jay M, Litt M, Craig I, Davies K (1990) Localization of the microsatellite probe DXS 426 between DXS 7 and DXS 225 on Xp and linkage to Xlinked retinitis pigmentosa. Am J Hum Genet 47:935-940

Hardcastle AJ, Thiselton AW, Van Maedergem L, Saha BK, Jay M, Plant C, Taylor R, Bird AC, Bhattacharya SS (1999) Mutations in the $R P 2$ gene cause disease in $10 \%$ of families with familial $\mathrm{X}$-linked retinitis pigmentosa assessed in this study. Am J Hum Genet 64:1210-1215

Mears AJ, Gieser L, Yan D, Chen C, Fahrner S, Hiriyanna S, Fujita R, Jacobson SG, Sieving PA, Swaroop A (1999) Protein truncation mutation in the $R P 2$ gene in a North American cohort of families with X-linked retinitis pigmentosa. Am J Hum Genet 64:897-900

Meindl A, Dry K, Herrmann K, Manson F, Ciccodicola A, Edgar A, Carvalho MRS, Achatz H, Hellebrand H, Lennon A, Migliaccio C, Porter K, Zrenner E, Bird A, Jay M, Lorenz B, Wittwer B, D'Urso M, Meitinger T, Wright A (1996) A gene (RPGR) with homology to the RCC1 guanine nucleotide exchange factor is mutated in $\mathrm{X}$-linked retinitis pigmentosa. Nat Genet 13:35-42

Schwahn U, Lenzner S, Dong J, Feil S, Hinzmann B, Duijnhoven G, Kirschner E, Hamberger M, Bergen AAB, Rosenberg T, Pinckers AJLG, Fundele R, Rosenthal A, Cremers FPM, Ropers H-H, Berger W (1998) Positional cloning of the gene for X-linked retinitis pigmentosa. Nat Genet 19:327-332

Shastry BS, Trese MT (1995) X-Linked retinitis pigmentosa: report of a large kindred with loss of central vision and preserved peripheral function. Am J Med Genet 59:401-402

Shastry BS, Hejtmancik JF, Plager DA, Hartzer MK, Trese MT (1995) Linkage and candidate gene analysis of X-linked familial exudative vitreoretinopathy. Genomics 27:341-344

Teague PW, Aldred MA, Jay M, Dempster M, Harrison C, Carothers AD, Hadrick LJ, Evans HJ, Strain L, Brock DJH, Bundey S, Jay B, Bird AC, Bhattacharya SS, Wright AF (1994) Heterogeneity analysis in 40 X-linked retinitis pigmentosa families. Am J Hum Genet 55:105-111 